\title{
Squish and squeeze - the nucleus as a physical barrier during migration in confining environments
}

\author{
Alexandra Lynn McGregor ${ }^{1,3 \dagger}$, Chieh-Ren $\mathrm{Hsia}^{2,3 \dagger}$ and Jan Lammerding ${ }^{1,3}$ \\ 1. Nancy C. and Peter E. Meinig School of Biomedical Engineering, Cornell University, Ithaca, NY 14853, USA. \\ 2. Department of Molecular Biology and Genetics, Cornell University, Ithaca, NY 14853, USA. \\ 3. Weill Institute for Cell and Molecular Biology, Cornell University, Ithaca, NY 14853, USA. \\ $\dagger$ Authors contributed equally. \\ Corresponding Author: \\ jan.lammerding@cornell.edu \\ Cornell University, Weill Hall, Room 235; 526 Campus Road; Ithaca, NY 14853
}

\begin{abstract}
From embryonic development to cancer metastasis, cell migration plays a central role in health and disease. It is increasingly becoming apparent that cells migrating in three-dimensional (3-D) environments exhibit some striking differences compared with their well-established 2-D counterparts. One key finding is the significant role the nucleus plays during 3-D migration: when cells move in confined spaces, the cell body and nucleus must deform to squeeze through available spaces, and the deformability of the large and relatively rigid nucleus can become rate-limiting. In this review, we highlight recent findings regarding the role of nuclear mechanics in 3-D migration, including factors that govern nuclear deformability, and emerging mechanisms by which cells apply cytoskeletal forces to the nucleus to facilitate nuclear translocation. Intriguingly, the 'physical barrier' imposed by the nucleus also impacts cytoplasmic dynamics that affect cell migration and signaling, and changes in nuclear structure resulting from the mechanical forces acting on the nucleus during 3-D migration could further alter cellular function. These findings have broad relevance to the migration of both normal and cancerous cells inside living tissues, and motivate further research into the molecular details by which cells move their nuclei, as well as the consequences of the mechanical stress on the nucleus.
\end{abstract}

\section{Introduction}

In multicellular organisms, cell migration is essential in the development, maintenance and repair of various tissues [1]; it also enables immune cells to survey tissues and to respond to local challenges [2]. At the same time, cell migration drives the tissue invasion and metastasis of cancer cells, which is responsible for the vast majority of cancer deaths [3]. While much of our current knowledge regarding the molecular and biophysical principles of cell migration stems from studying cells moving on 2-D substrates [4], it is now becoming evident that cells migrating in 3-D environments encounter distinct physical challenges. During in vivo migration/invasion, cells must navigate many microstructural obstacles, including extracellular matrix (ECM) networks and neighboring cells. The pore sizes encountered in the interstitial space range from 0.1 to $30 \mu \mathrm{m}$ in diameter, i.e., comparable to or significantly smaller than the size of the migrating cell [5-7]. Cells have two strategies to penetrate such confining environments: (i) expanding the openings via physical remodeling and/or proteolytic degradation of the ECM [8], or (ii) contorting their shape to accommodate the available spaces [9]. The cell membrane and cytoplasm are able to quickly deform and remodel to penetrate openings less than $1 \mu \mathrm{m}$ in diameter [10]. In contrast, deformation of the nucleus, the largest and stiffest organelle, presents a more formidable challenge. Here we discuss emerging insights into the intracellular biomechanics and molecular processes involved in translocating the nucleus through tight spaces, including implications on migration efficiency and other biological functions. 


\section{The size and rigidity of the nucleus: a physical barrier for cell migration}

The nucleus is the largest organelle in the cell, with a diameter between 3-15 $\mu \mathrm{m}[11,12]$, making it substantially larger than many pores encountered during migration in physiological tissues. Furthermore, biophysical measurements of isolated nuclei and intact cells reveal that the nucleus is typically 2- to 10-times stiffer than the surrounding cytoplasm [11]. This combination of large size and relative rigidity of the nucleus led to the hypothesis that the nucleus can impact the cells' ability to migrate [13]. Early support for this hypothesis came from work on tumor cells migrating through microfabricated channels with precisely defined constrictions [14-16] (see Box 1 for more information on such devices). While moderate confinement results in increased migration speed by allowing cells to employ faster migration modes (e.g., 'amoeboid migration' and 'chimneying') than during 2-D migration [17], constrictions below approximately $5 \mu \mathrm{m}$ in diameter require substantial nuclear deformation and result in reduced migration speeds [14-16,18-20]. A seminal study by Friedl, Wolf, and colleagues using a range of cell types demonstrated that nuclear deformability presents a physical limit for the migration through collagen matrices with varying pore sizes [10]. When inhibiting matrix metalloprotease (MMP) activity required to degrade ECM, migration speed declined with decreasing pore size as nuclei had to undergo increasing deformation [10]. At pore sizes smaller than $10 \%$ of the non-deformed cross-section of the nucleus, cells reached a 'nuclear deformation limit' resulting in complete migration arrest, despite continued protrusion of the cytoplasm [10]. Subsequent studies using a variety of cell lines and experimental assays ranging from microfluidic devices, membranes with defined pores, ECM matrices, and in vivo xenografts have painted a similar picture, in which the deformability of the nucleus limits the cell's ability to pass through tight spaces, reducing or even stalling migration as the pore size decreases below the cross-section of the nucleus [18-26]. Assessing the role of specific physical factors on cell migration in confined environments, Lautscham and colleagues [20] found that increased nuclear (but not cytoplasmic) volume, increased nuclear stiffness, reduced cell adhesion and lower cell contractility impaired migration through microfluidic constrictions. While the above findings prove common to a large variety of cell lines, including neutrophils, fibroblasts, and tumor cells, the exact degree of confinement necessary to elicit such effects, and the magnitude of the effect, varies with cell type. These differences indicate that variation in nuclear deformability, or the cytoskeletal forces applied to the nucleus, may be important modulators of the 'nuclear barrier' effect.

\section{Lamins determine nuclear deformability and migration through confined environments}

The deformability of the nucleus is largely determined by two components, the nuclear lamin network and chromatin [27,28]. Lamins are type $\mathrm{V}$ nuclear intermediate filaments that can be divided into two sub-types, A-type (A, C, C2) and B-type (B1-3) lamins [29-36]. The different lamin subtypes form separate but interdigitating fibrillar networks at the nuclear periphery $[37,38]$. In addition to regulating nuclear shape and stiffness [27,39-42], they play important roles in chromatin organization, DNA damage repair, and transcriptional regulation $[37,43,44]$. Cell-stretching and micropipette aspiration experiments indicate that A-type lamins have a larger impact on nuclear stiffness than B-type lamins-nuclear stiffness strongly scales with expression of lamins A/C [39-42], although increased expression of lamin B1 can also increase nuclear rigidity [45]. Consistent with the 'nuclear barrier' hypothesis, recent studies found that cells with reduced levels of lamins $A / C$ have more deformable nuclei and migrate faster through tight spaces than control cells with normal lamin $A / C$ levels $[18,46]$. Conversely, increased expression of lamin A, or expression of a mutant lamin (progerin) that increases nuclear stiffness, impaired transit through narrow constrictions [21,25]. Loss of lamin A/C promotes cell migration through small constrictions by allowing larger nuclear deformation, rather than increased nuclear compression, as the nuclear volume does not decrease during nuclear 
translocation [19]. These findings have direct physiological and clinical relevance, since downregulation of lamins $A / C$ during granulopoiesis is critical for the ability of neutrophils to pass through micron-sized constrictions [21], and misregulation of lamins is common to many cancers [47] (see Box 2 for more information). Less is known about the role of B-type lamins in 3-D migration. Loss of B-type lamins impairs migration of neurons, which lack A-type lamins, in the developing brain, and this effect is thought to be caused by defects connecting the nuclear interior and cytoplasm [48,49]. Given recent reports that lamin A/C levels and organization can vary in response to substrate stiffness and cytoskeletal tension [41,50,51], it is intriguing to speculate that cells could also dynamically adjust their nuclear stiffness during migration.

\section{The role of chromatin in nuclear deformability and migration}

Chromatin, consisting of DNA wrapped around histone octamers, occupies most of the nuclear interior and contributes to the viscoelastic response of nuclear deformation [28,52]. Chromatin exists in two configurations: (i) open 'euchromatin', which is typically transcriptionally active, and (ii) closed, more compact 'heterochromatin', which is associated with inactive genes [53]. Promoting euchromatin over heterochromatin organization, for example by treatment with the deacetylase inhibitor trichostatin A (TSA), results in softer and more deformable nuclei [52]. Interestingly, treatment with 5'-deoxy-5'-methylthioadenosine (MTA), a methyltransferase inhibitor that cause de-condensation of chromatin, impairs the migration of the cells through microchannels [15]. It remains unclear whether this counterintuitive effect was due to the increase in nuclear size resulting from chromatin de-condensation, which may counteract reduced nuclear stiffness [20], or due to altered transcriptional regulation, motivating future research on the role of chromatin organization in cell migration through confining constrictions.

\section{Cytoskeletal forces pulling or pushing on the nucleus}

Recent studies have shed light onto the molecular components physically connecting the nucleus and cytoskeleton (Fig. 1A), revealing that the linker of nucleoskeleton and cytoskeleton (LINC) complex is the 'clutch' that enables transmission of mechanical force across the nuclear envelope [54]. We refer the reader to excellent recent reviews $[55,56]$ and the article by Burridge et al. in this issue for more details on LINC complex function. However, the mechanism by which cells move the nucleus through confining constrictions, including whether cells predominantly pull or push on the nucleus (Fig. 1B), remains incompletely understood. It is likely that cells apply a varying combination of both mechanisms, depending on the specific conditions.

\section{Pulling the nucleus forward}

In most migrating cells, the nucleus is positioned rearward, i.e., in the back of the cell [55]. Recent studies indicate that actomyosin contractility, possibly in combination with intermediate filaments [57,58], physically pull the nucleus forward during 2-D and 3-D migration $[10,59]$. In 3-D lamellipodial-based migration, actomyosin contractility and integrin-mediated traction at the leading edge are required to translocate the nucleus through narrow constrictions [10]. During 3-D lobopodial-based migration, non-muscle myosin IIA (NMIIA)-containing actomyosin bundles work with vimentin filaments to pull the nucleus forward by binding to nesprin-3a of the LINC complex via plectin $[58,60]$. Whereas microtubule associated motors are dispensable for nuclear translocation in 2-D migration [59], the microtubule-associated motors dynein/kinesin, which can directly bind to LINC complex proteins [56], are essential for interkinetic nuclear migration in neurons [61]. Dynein and kinesis are also required for nuclear positioning in multinucleated myotubes, where nuclei are squeezed, rotated and translocated to form proper myotube structure [62]. Thus, it is possible that microtubule-associated motors also participate in nuclear translocation during 3D cell migration. 


\section{Pushing the nucleus forward}

Actomyosin contraction at the back of the cell is required for squeezing the leukocyte nucleus through narrow pores [63]. In breast and brain cancer cells, non-muscle myosin IIB (NMIIB) is recruited to the perinuclear cytoskeleton and posterior of the cell, possibly via nesprin-2, where it applies pushing forces to the nucleus to facilitate nuclear translocation through confining 3-D environments $[64,65]$. Depletion of NMIIB, but not NMIIA, impairs migration of breast cancer cells through microfluidic constrictions and dense collagen matrices [64], suggesting a specific role of perinuclear NMIIB actin networks in squeezing the nucleus through constrictions. Nonetheless, further studies are required to determine whether NMIIB-dependent actomyosin contraction constitutes a general mechanism for nuclear translocation, whether it also involves nesprin-3 $\alpha$ and vimentin filaments, and whether increased pulling forces can compensate for impaired pushing forces and vice versa.

\section{Physical compartmentalization by the nucleus}

During migration through confined spaces, the nucleus takes up most of the space within the constriction, thus leaving little or no room for cytoplasmic transport around the nucleus and effectively dividing the cytoplasm into "front" and "back" compartments (Fig. 1B). This intracellular compartmentalization plays an important role in two mechanisms: osmotic pressure difference caused by water permeation, and intracellular pressure generated by the nucleus as an active 'piston' $[60,66]$. Polarized distribution of $\mathrm{Na}^{+} / \mathrm{H}^{+}$pumps and aquaporins on the plasma membrane at the front and back of the cell alters water permeation and can drive migration and nuclear translocation, even when actin polymerization is inhibited [66]. However, since this osmotic pressure-based mechanism has only been described in tube-like microchannels that restrict fluid flow to the front and back of the cells, its relevance in more physiological environments still needs to be examined. Work by the Yamada group [60] reveals that during lobopodial-based migration, actomyosin-mediated pulling of the nucleus compresses the front of the cell, similar to a piston in a cylinder, building up higher intracellular pressure within this compartment and driving the formation of new lobopodial protrusions at the leading edge. The nucleus may similarly serve as a counterbalance for the directional protrusion of invadopodia, causing visible indentation of the basal nuclear surface [67]. Taken together, these studies illustrate how cells can take advantage of the large and rigid nucleus to promote cell migration in 3-D environments.

\section{Biological consequences of nuclear deformation during 3-D cell migration}

The nucleus is not only a passive mechanical element, but also houses the cell's genomic information and is the site of DNA replication, transcription, and RNA processing. Thus, the substantial mechanical forces and deformation incurred during migration through tight spaces may have severe biological consequences that impact cellular function and viability.

\section{Influences on cell survival and genomic stability}

Recent studies have produced conflicting results on the effect of cell migration through tight spaces on cell viability. Whereas one study found increased apoptosis after migration through small $(3 \mu \mathrm{m})$ pores, particularly in lamin A/C-deficient cells [46], others reported no noticeable increase in cell death during confinement $[17-19,21]$. These apparent differences may reflect differences in cell type or mechanical confinement (e.g., porous membranes vs. microfluidic devices and collagen matrices). Interestingly, pharmacological inhibition of the heat shock protein 90 (HSP90), which facilitates DNA damage repair [68], results in increased cell death after passage through 3- $\mu$ m diameter pores, suggesting that cells may have suffered DNA damage during migration that causes apoptosis when not adequately repaired [46]. Mechanical 
stress alone can induce DNA damage [69], but it remains to be seen whether the mechanical stress incurred during 3-D cell migration is sufficient to cause DNA damage.

\section{Influences on mechanotransduction signaling and gene expression}

Mechanical stress on the nucleus during migration may also trigger non-lethal changes, which could further affect cell migration and cellular function. Recent studies in which isolated nuclei were subjected to mechanical stress suggest that the nucleus itself can act as a mechanosensitive element. Force application via the LINC complex induces rapid phosphorylation of the inner nuclear membrane protein emerin, leading to recruitment of lamin $\mathrm{A} / \mathrm{C}$ to the nuclear envelope and nuclear stiffening [70]. Shearing isolated nuclei causes partial unfolding of lamin $\mathrm{A} / \mathrm{C}$, exposing cryptic binding sites that could initiate mechanotransduction events [41]. Furthermore, changes in the mechanical microenvironment and force application to intact cells can induce chromatin remodeling [71,72] and dissociation of nuclear protein complexes [73], which could affect both nuclear deformability and gene expression (see [74] and [75] for a detailed discussion of nuclear mechanotransduction). While these reports suggest that nuclear deformation during 3-D migration could impact nuclear organization, chromatin remodeling, and gene expression, direct experimental evidence for this hypothesis is still missing.

\section{Conclusions and Outlook}

The nucleus has long been recognized as a central hub for genomic information and its processing. Work published over the last few years makes it clear that one has to also consider the physical impact of the nucleus on cellular function, particularly during migration in 3-D environments. The nucleus, with its large volume and relative rigidity, acts as physical barrier when cells encounter pore sizes smaller than the nuclear diameter, resulting in reduced migration efficiency or even complete migration arrest. The extent of this nuclear barrier effect is largely driven by the nuclear size and stiffness, which is governed by the levels of the nuclear envelope proteins lamin $\mathrm{A} / \mathrm{C}$ and chromatin organization. These findings are particularly relevant to immune cells and to invading cancer cells, which move through tissues with pore sizes smaller than the size of the nucleus and often have altered expression of lamins and other nuclear envelope proteins. Despite recent advances, many open questions remain. For example, can cells dynamically adjust their nuclear stiffness to facilitate cell migration through tight spaces, possibly by phosphorylation and/or degradation of nuclear lamins? Are some cells particularly well-suited for 3-D migration, either by having more deformable nuclei or by pulling/pushing harder on the nucleus? What are the precise molecular mechanisms by which cells translocate the nucleus through tight constrictions? And what are the biological consequences resulting from the large nuclear deformations, which could include changes in chromatin organization, DNA damage, and altered gene expression? Such mechanically induced events could not only affect migration itself, e.g., by altering nuclear stiffness and cytoskeletal dynamics, but also impact various other cellular functions and even viability. To drive new discoveries, it will be crucial to combine new imaging tools, such as fluorescence resonance energy transfer (FRET)-based intracellular force probes [76], with microfabricated environments that mimic physiological environments while providing defined geometries and enhanced live-cell imaging conditions, as well as single cell based assays to measure cell viability, gene expression, and epigenetic modification. Further insights into the role of the nucleus in 3-D migration will not only improve our understanding of the physical constraints during migration in physiological environments, but may ultimately lead to new strategies to better target invasive cancer cells and to reduce or eliminate metastatic spreading. 


\section{Acknowledgments}

The authors apologize to all authors whose work could not be cited due to space constraints. The authors thank Emily Bell and Peter DelNero for discussion and critical reading of the manuscript. This work was supported by awards from the National Institutes of Health [R01 HL082792], the National Science Foundation [CBET-1254846], the National Cancer Institute [U54CA143876], and the Department of Defense [Breast Cancer Idea Award BC102152], as well as a NSF Graduate Research Fellowship to ALM [DGE-1144153]. The content of this article is solely the responsibility of the authors and does not necessarily represent the official views of the National Cancer Institute or the National Institutes of Health.

\section{Boxes}

\section{Box 1: Development of tools to study migration in confined environments.}

Microfabrication techniques are finding increased application to study cell migration in confined environments, complementing existing approaches such as transwell plates and collagen invasion assays $[10,46,77,78]$. Besides micropillars, polymer scaffolds, and electrospun matrices $[25,26,79,80]$, microfluidic devices made from polydimethylsiloxane (PDMS) by soft lithography $[81,82]$ have proven particularly powerful in investigating cell migration through tight spaces by providing precisely defined microscale structures and constrictions with crosssections from $100 \mu^{2}$ to less than $5 \mu m^{2}$ [15-21,23,24,64,66,83]. These devices, which often include features to apply stable chemotactic gradients [14-16,18,19,24], allow for user defined geometries ranging from simple straight channels $[15,16,24,66]$ to more intricate designs mimicking physiological environments $[18-21,23,83]$. The migration devices can be functionalized with a variety of ECM proteins to control cell adhesion. Since the devices are made of transparent PDMS and mounted on thin coverslips, they provide superb imaging conditions for live-cell imaging with high spatial and temporal resolution $[81,82]$.

\section{Box 2: Lamins, nuclear shape, and disease}

Mutations in lamins cause a large spectrum of human diseases, ranging from muscular dystrophy and dilated cardiomyopathy to premature aging $[84,85]$. Increasing reports indicate that altered lamin expression (rather than mutations) is found in many cancers, and often correlates with negative clinical outcomes $[47,86,87]$. For example, reduced expression of lamin $\mathrm{A} / \mathrm{C}$ has been reported in breast [88,89] and cervical cancer [90], and is correlated with an increased recurrence of stage II and III colon cancer [91] and reduced disease free survival in breast cancer [89]. However, in other cases, increased A-type lamin expression is associated with disease progression, specifically in prostate, colon and ovarian cancers [92,93]. Given the multiple function of lamins, changes in their expression are expected to have pleiotropic effects, affecting not only nuclear stiffness but also proliferation, survival, and gene expression $[47,86,87,93,94]$. Nonetheless, it is intriguing that more invasive breast cancer cell lines such as Hs578T and MDA-MB-231 are capable of more extensive nuclear deformation than normal and non-malignant controls [95], and move faster through migration devices designed to mimic tight spaces inside the body [15]. Future studies should be directed at characterizing the effects of altered lamin levels on cell migration, as well as other cellular functions, in more detail. 


\section{Table}

\begin{tabular}{|c|c|c|}
\hline Components & Functions & References \\
\hline Lamins & $\begin{array}{l}\text { 1. Major components of the nuclear lamina; determine } \\
\text { nuclear shape and size; A-type lamins in particular } \\
\text { are major contributors to nuclear stiffness. } \\
\text { 2. Contribute to anchoring LINC complex at the nuclear } \\
\text { envelope } \\
\text { 3. Bind to chromatin, particularly at lamin-associated } \\
\text { domains (LADs) at the nuclear periphery; also } \\
\text { interact with transcriptional regulators and DNA } \\
\text { damage response proteins. }\end{array}$ & $\begin{array}{l}{[25][37][39][40]} \\
{[54]}\end{array}$ \\
\hline Chromatin & $\begin{array}{l}\text { 1. Contributes to nuclear stiffness } \\
\text { 2. Rearrangement can regulate gene expression in } \\
\text { response to nuclear deformation }\end{array}$ & [28] [52] [96] [97] \\
\hline LINC complexes & $\begin{array}{l}\text { 1. Physically connect the cytoskeleton and the nucleus, } \\
\text { enabling force application and relaying of mechanical } \\
\text { signal to the nucleus }\end{array}$ & [54] [55] [56] \\
\hline $\begin{array}{l}\text { Actomyosin bundles } \\
\text { and non-muscle myosin } \\
\text { II (NMII) }\end{array}$ & $\begin{array}{l}\text { 1. Actomyosin contraction is the major player of both } \\
\text { actively pulling and pushing the nucleus } \\
\text { 2. Actomyosin contraction is crucial for relaying } \\
\text { mechanical signal to the nucleus } \\
\text { 3. Non-muscle myosin IIA participates in actomyosin } \\
\text { contraction at cell anterior to pull the nucleus. } \\
\text { 4. Non-muscle myosin IIB participates in actomyosin } \\
\text { contraction at cell posterior to push the nucleus. }\end{array}$ & $\begin{array}{l}{[10][57][60][63]} \\
{[64]}\end{array}$ \\
\hline Intermediate filaments & $\begin{array}{l}\text { 1. Vimentin filaments interact with actomyosin (NMIIA) } \\
\text { bundles to facilitate pulling of the nucleus } \\
\text { 2. Have the highest resistance to tensile force among } \\
\text { the cytoskeleton, providing the strength needed for } \\
\text { pulling the nucleus }\end{array}$ & [60] [98] \\
\hline Integrins & $\begin{array}{l}\text { 1. Form focal adhesion at the leading edge of the cell, } \\
\text { whose traction mediated by integrin is needed for } \\
\text { nuclear translocation }\end{array}$ & [10] [99] \\
\hline $\begin{array}{l}\text { Microtubules and } \\
\text { dynein/kinesin motor } \\
\text { proteins }\end{array}$ & $\begin{array}{l}\text { 1. Participate in nuclear rotation during } 2 D \text { migration } \\
\text { and in myotubes } \\
\text { 2. Possible role in nuclear rotation and pulling in } 3 D \\
\text { migration }\end{array}$ & [55] [62] [100] \\
\hline
\end{tabular}

Table 1. Overview of relevant molecular components involved in nuclear mechanics and migration in confining environments. 


\section{Figure legend}

Figure 1. Schematic overview of the physical connections between the nucleus and cytoskeleton, and their roles in moving the nucleus through confined spaces. (A) At the nuclear periphery, chromatin interacts with lamins at lamina-associated domains (LADs). SUN domain proteins (SUN1 and SUN2) are anchored to the nuclear lamina and other components of the nuclear interior by their $\mathrm{C}$-terminus. The $\mathrm{N}$-terminal luminal long stalks and SUN domains of SUN1/2 form trimers that interact with $\mathrm{KASH}$ domain proteins located in the outer nuclear membrane (nesprin-1/-2/-3, along with the cell-type specific nesprin-4 and KASH5), forming the LINC complex [101]. The strong interaction between SUN domain trimers and the KASH domains provide the basis to mechanically couple the nuclear interior with the cytoskeletonnesprins interact directly with actomyosin bundles, or indirectly with microtubules and intermediate filaments via intermediary proteins (such as kinesin, dynein, plectin) [55]. Mechanical force transmission via nucleo-cytoskeletal coupling may also trigger mechanotransduction events, ranging from the recruitment of lamins to the LINC complex to changes in chromatin organization and gene expression, which may further impact cell migration processes. (B) Cytoskeletal organization and dynamics during migration in confined 3$\mathrm{D}$ environments. As the cell passes through narrow pores, the nucleus separates the cell into front and back ends compartments. The insets depict proposed mechanisms by which the cytoskeleton translocates the nucleus through confined spaces, including pushing via actomyosin contraction at the posterior of the nucleus (1), pulling via actomyosin contraction facilitated by intermediate filaments (2), pulling via microtubule-associated motors (3), and rotation via microtubule-associated motors (4). 


\section{References and recommended readings}

1. Munjal A, Lecuit T: Actomyosin networks and tissue morphogenesis. Development 2014, 141:1789-93.

2. Weninger W, Biro $M$, Jain R: Leukocyte migration in the interstitial space of nonlymphoid organs. Nat. Rev. Immunol. 2014, 14:232-46.

3. Chaffer CL, Weinberg $\mathrm{R}$ a: A perspective on cancer cell metastasis. Science 2011 , 331:1559-64.

4. Gardel ML, Schneider IC, Aratyn-Schaus Y, Waterman CM: Mechanical integration of actin and adhesion dynamics in cell migration. Annu. Rev. Cell Dev. Biol. 2010, 26:315-33.

5. Doerschuk CM, Beyers N, Coxson HO, Wiggs B, Hogg JC: Comparison of neutrophil and capillary diameters and their relation to neutrophil sequestration in the lung. $J$. Appl. Physiol. 1993, 74:3040-5.

6. Stoitzner P, Pfaller K, Stössel H, Romani N: A close-up view of migrating Langerhans cells in the skin. J. Invest. Dermatol. 2002, 118:117-25.

7. Weigelin B, Bakker G-J, FriedI P: Intravital third harmonic generation microscopy of collective melanoma cell invasion. IntraVital 2012, 1:32-43.

8. Stetler-Stevenson WG, Aznavoorian S, Liotta LA: Tumor cell interactions with the extracellular matrix during invasion and metastasis. Annu. Rev. Cell Biol. 1993, 9:541-73.

9. Wolf K, Mazo I, Leung H, Engelke K, Von Andrian UH, Deryugina El, Strongin AY, Bröcker EB, Friedl $P$ : Compensation mechanism in tumor cell migration:

Mesenchymal-amoeboid transition after blocking of pericellular proteolysis. J. Cell Biol. 2003, 160:267-77.

${ }^{* *} 10$. Wolf K, te Lindert M, Krause M, Alexander S, te Riet J, Willis a. L, Hoffman RM, Figdor CG, Weiss SJ, Friedl P: Physical limits of cell migration: Control by ECM space and nuclear deformation and tuning by proteolysis and traction force. J. Cell Biol. 2013, 201:1069-84.

This is the first study to directly demonstrate the concept of the nucleus as a physical barrier during 3-D cell migration, including in physiological collagen networks. Using a variety of cell lines, the authors show that when proteolytic ECM degradation is inhibited, cell migration speed through collagen matrices decreases with decreasing pore size, and cell migration is completely arrested when cell reach a 'nuclear deformability limit'.

11. Lammerding J: Mechanics of the nucleus. Compr. Physiol. 2011, 1:783-807.

12. Martins RP, Finan JD, Guilak F, Lee D a: Mechanical regulation of nuclear structure and function. Annu. Rev. Biomed. Eng. 2012, 14:431-55.

13. Friedl $\mathrm{P}$, Wolf K, Lammerding J: Nuclear mechanics during cell migration. Curr. Opin. Cell Biol. 2011, 23:55-64.

*14. Tong Z, Balzer EM, Dallas MR, Hung WC, Stebe KJ, Konstantopoulos K: Chemotaxis of cell populations through confined spaces at Single-Cell resolution. PLoS One 2012, 7:1-10. 
This was one of the first studies to use a microfluidic device, including a self-sustaining chemotactic gradient, to study cell migration on a single-cell level. The device enabled the authors to test the effect of various extracellular matrix coatings applied to the microfluidic channels, as well as to deliver different phramcological drugs to the cells and to monitor the consequences on migration.

*15. Fu Y, Chin LK, Bourouina T, Liu AQ, VanDongen AMJ: Nuclear deformation during breast cancer cell transmigration. Lab Chip 2012, 12:3774-8.

This was the first study to assess the effect of a disease-causing lamin A mutation on cell migration through confining environments. The authors studied cells expressing progerin, the mutant form of lamin A responsible for Hutchinson-Gilford progeria syndrome, moving through an array of PDMS micropillars with decreasing spacing. Progerin expressing cells, which have stiffer nuclei, were were less motile, and eventually incapable of migrating through the decreasing spaces between the pillars, compared to normal control cells.

16. Balzer EM, Tong Z, Paul CD, Hung WC, Stroka KM, Boggs AE, Martin SS, Konstantopoulos K: Physical confinement alters tumor cell adhesion and migration phenotypes. FASEB J. 2012, 26:4045-56.

17. Liu Y-J, Le Berre M, Lautenschlaeger F, Maiuri P, Callan-Jones A, Heuzé M, Takaki T, Voituriez R, Piel M: Confinement and Low Adhesion Induce Fast Amoeboid Migration of Slow Mesenchymal Cells Cell 2015, 160:659-72.

**18. Davidson PM, Denais C, Bakshi MC, Lammerding J: Nuclear Deformability Constitutes a Rate-Limiting Step During Cell Migration in 3-D Environments. Cell. Mol. Bioeng. 2014, 7:293-306.

This study provides a detailed characterization of the effects of different levels of lamin $\mathrm{A} / \mathrm{C}$ on migration through confined spaces. The authors examine lamin $\mathrm{A} / \mathrm{C}$-deficient and wild-type mouse embryo fibroblasts migrating through a novel microfluidic devices with precisely defined constrictions, finding that while in wild-type cells migration speed decreases when the cells encounter constrictions smaller than $25 \mu \mathrm{m} 2$, cells completley lacking lamins $\mathrm{A} / \mathrm{C}$ show no reduction in normalized migration speed, and heterozygous cells with $50 \%$ of normal lamin $\mathrm{A} / \mathrm{C}$ levels show an intermediate phenotype.

19. Davidson PM, Sliz J, Isermann P, Denais CM, Lammerding J: Design of a microfluidic device to quantify dynamic intra- nuclear deformation during cell migration through confining environments. Integr. Biol. 2015, 7: 1534-46.

*20. Lautscham LA, Kämmerer C, Lange JR, Kolb T, Mark C, Schilling A, Strissel PL, Strick R, Gluth $C$, Rowat AC, et al.: Migration in Confined 3D Environments Is Determined by a Combination of Adhesiveness, Nuclear Volume, Contractility, and Cell Stiffness. Biophys. J. 2015, 109:900-13.

This study clearly provides a detailed characterization of multiple physical factors contributing to efficient 3-D cell migration in confined environments. Using microfluidic constriction channels and collagen matrix assays with multiple cell lines, the authors show that the velocity of cell migration through confining constrictions depends not only on cell stiffness, but also on nuclear volume, cell adhesiveness and contractility.

*21. Rowat AC, Jaalouk DE, Zwerger M, Ung WL, Eydelnant IA, Olins DE, Olins AL, Herrmann H, Weitz DA, Lammerding J: Nuclear Envelope Composition Determines the Ability of Neutrophil-type Cells to Passage through Micron-scale Constrictions. 


\section{J. Biol. Chem. 2013, 288:8610-8.}

This study provided the first functional evidence that the downregulation of lamin $\mathrm{A} / \mathrm{C}$ during granulopoiesis facilitates the transit of neutrophils in micron-sized constrictions during perfusionand migration, highlighting the physiological relevance of nuclear deformability and changes in lamin levels.

22. Guzman A, Ziperstein MJ, Kaufman LJ: The effect of fibrillar matrix architecture on tumor cell invasion of physically challenging environments. Biomaterials 2014, 35:6954-63.

23. Mak M, Reinhart-King $\mathrm{C}$ a, Erickson D: Elucidating mechanical transition effects of invading cancer cells with a subnucleus-scaled microfluidic serial dimensional modulation device. Lab Chip 2013, 13:340-8.

24. Malboubi M, Jayo A, Parsons M, Charras G: An open access microfluidic device for the study of the physical limits of cancer cell deformation during migration in confined environments. Microelectron. Eng. 2015, 144:42-5.

*25. Booth-Gauthier E a, Du V, Ghibaudo M, Rape AD, Dahl KN, Ladoux B: HutchinsonGilford progeria syndrome alters nuclear shape and reduces cell motility in three dimensional model substrates. Integr. Biol. 2013, 5:569-77.

This was the first study to assess the effect of a disease-causing lamin A mutation on cell migration through confining environments. The authors studied cells expressing progerin, the mutant form of lamin A responsible for Hutchinson-Gilford progeria syndrome, moving through an array of PDMS micropillars with decreasing spacing. Progerin expressing cells, which have stiffer nuclei, were were less motile, and eventually incapable of migrating through the decreasing spaces between the pillars, compared to normal control cells.

26. Greiner AM, Jäckel M, Scheiwe AC, Stamow DR, Autenrieth TJ, Lahann J, Franz CM, Bastmeyer M: Multifunctional polymer scaffolds with adjustable pore size andchemoattractant gradients for studying cell matrix invasion. Biomaterials 2014, 35:611-9.

27. Dahl KN, Kahn SM, Wilson KL, Discher DE: The nuclear envelope lamina network has elasticity and a compressibility limit suggestive of a molecular shock absorber. $J$. Cell Sci. 2004, 117:4779-86.

28. Dahl KN, Engler AJ, Pajerowski JD, Discher DE: Power-Law Rheology of Isolated Nuclei with Deformation Mapping of Nuclear Substructures. Biophys. J. 2005, 89:2855-64.

29. Fisher DZ, Chaudhary N, Blobel G: cDNA sequencing of nuclear lamins A and C reveals primary and secondary structural homology to intermediate filament proteins. Proc. Natl. Acad. Sci. U. S. A. 1986, 83:6450-4.

30. McKeon FD, Kirschner MW, Caput D: Homologies in both primary and secondary structure between nuclear envelope and intermediate filament proteins. Nature 1986, 319:463-8.

31. Furukawa $\mathrm{K}$, Inagaki $\mathrm{H}$, Hotta $\mathrm{Y}$ : Identification and cloning of an mRNA coding for a germ cell-specific A-type lamin in mice. Exp. Cell Res. 1994, 212:426-30.

32. Peter M, Kitten GT, Lehner CF, Vorburger K, Bailer SM, Maridor G, Nigg E a: Cloning and sequencing of cDNA clones encoding chicken lamins $A$ and $B 1$ and 
comparison of the primary structures of vertebrate A- and B-type lamins. J. Mol. Biol. 1989, 208:393-404.

33. Vorburger K, Lehner CF, Kitten GT, Eppenberger HM, Nigg EA: A second higher vertebrate B-type lamin. cDNA sequence determination and in vitro processing of chicken lamin B2. J. Mol. Biol. 1989, 208:405-15.

34. Lin F, Worman HJ: Structural organization of the human gene (LMNB1) encoding nuclear lamin B1. Genomics 1995, 27:230-6.

35. Lin F, Worman HJ: Structural organization of the human gene encoding nuclear lamin A and nuclear lamin C. J. Biol. Chem. 1993, 268:16321-6.

36. Machiels BM, Zorenc AHG, Endert JM, Kuijpers HJH, Van Eys GJJM, Ramaekers FCS, Broers $\mathrm{JL}$ V: An alternative splicing product of the lamin A/C gene lacks exon 10. J. Biol. Chem. 1996, 271:9249-53.

37. Shimi T, Pfleghaar K, Kojima S, Pack C-G, Solovei I, Goldman AE, Adam SA, Shumaker DK, Kinjo M, Cremer T, et al.: The A- and B-type nuclear lamin networks:

microdomains involved in chromatin organization and transcription. Genes Dev. 2008, 22:3409-21.

38. Shimi T, Kittisopikul M, Tran J, Goldman AE, Adam SA, Zheng Y, Jaqaman K, Goldman RD: Structural organization of nuclear lamins A, C, B1, and B2 revealed by superresolution microscopy. Mol. Biol. Cell 2015, 26:4075-86.

39. Lammerding J, Schulze PC, Takahashi T, Kozlov S, Sullivan T, Kamm RD, Stewart CL, Lee RT: Lamin A/C deficiency causes defective nuclear mechanics and mechanotransduction. J. Clin. Invest. 2004, 113:370-8.

40. Lammerding J, Fong LG, Ji JY, Reue K, Stewart CL, Young SG, Lee RT: Lamins a and C but not lamin B1 regulate nuclear mechanics. J. Biol. Chem. 2006, 281:25768-80.

41. Swift J, Ivanovska IL, Buxboim A, Harada T, Dingal PCDP, Pinter J, Pajerowski JD, Spinler KR, Shin J-W, Tewari M, et al.: Nuclear lamin-A scales with tissue stiffness and enhances matrix-directed differentiation. Science 2013, 341:1240104.

42. Schäpe J, Prausse S, Radmacher M, Stick R: Influence of lamin A on the mechanical properties of amphibian oocyte nuclei measured by atomic force microscopy. Biophys. J. 2009, 96:4319-25.

43. Ho CY, Lammerding J: Lamins at a glance J. Cell Sci. 2012, 125:2087-93.

44. Solovei I, Wang AS, Thanisch K, Schmidt CS, Krebs S, Zwerger M, Cohen T V., Devys $\mathrm{D}$, Foisner $\mathrm{R}$, Peichl $\mathrm{L}$, et al.: LBR and lamin A/C sequentially tether peripheral heterochromatin and inversely regulate differentiation. Cell 2013, 152:584-98.

45. Ferrera D, Canale C, Marotta R, Mazzaro N, Gritti M, Mazzanti M, Capellari S, Cortelli P, Gasparini L: Lamin B1 overexpression increases nuclear rigidity in autosomal dominant leukodystrophy fibroblasts. FASEB J. Off. Publ. Fed. Am. Soc. Exp. Biol. 2014, 28:3906-18.

${ }^{* *}$ 46. Harada T, Swift J, Irianto J, Shin JW, Spinler KR, Athirasala A, Diegmiller R, Dingal PCDP, Ivanovska IL, Discher DE: Nuclear lamin stiffness is a barrier to 3D migration, but softness can limit survival. J. Cell Biol. 2014, 204:669-82.

This is the first study to demonstrate that while reduced levels of lamin $A / C$ can promote 
migration through narrow constrictions, the increased nuclear deformability/fragility makes cells more sensitive to migration-induced mechanical stress. Using fibronectincoated transwell assays and mouse xenografts, the authors show that intermediate A:Btype lamin ratio promotes cell migration both in vitro and in vivo, while deep knockdown of A-type lamin results in increased apoptosis in cells migrating through 3 - $\mu$ m diameter pores, which can be partially rescued by treatment with an HSP90 inhibitor.

*47. Hutchison CJ: Do lamins influence disease progression in cancer? Adv. Exp. Med. Biol. 2014, 773:593-604.

The author provides a comprehensive review that summarizes and compares recent findings of altered lamin expression in a wide variety of cancers, while also discussing potential mechanisms by which lamins may impact cancer progression and metastasis.

48. Coffinier C, Chang SY, Nobumori C, Tu Y, Farber E a, Toth JI, Fong LG, Young SG: Abnormal development of the cerebral cortex and cerebellum in the setting of lamin B2 deficiency. Proc. Natl. Acad. Sci. U. S. A. 2010, 107:5076-81.

49. Young SG, Jung H-J, Lee JM, Fong LG: Nuclear Lamins and Neurobiology. Mol. Cell. Biol. 2014, 34:2776-85.

50. Ihalainen TO, Aires L, Herzog FA, Schwartlander R, Moeller J, Vogel V: Differential basal-to-apical accessibility of lamin $\mathrm{A} / \mathrm{C}$ epitopes in the nuclear lamina regulated by changes in cytoskeletal tension. Nat. Mater. 2015, 14:1252-61.

51. Buxboim A, Swift J, Irianto J, Spinler KR, Dingal PCDP, Athirasala A, Kao Y-RC, Cho S, Harada T, Shin J-W, et al.: Matrix Elasticity Regulates Lamin-A,C Phosphorylation and Turnover with Feedback to Actomyosin. Curr. Biol. 2014, 24:1909-17.

52. Pajerowski JD, Dahl KN, Zhong FL, Sammak PJ, Discher DE: Physical plasticity of the nucleus in stem cell differentiation. Proc. Natl. Acad. Sci. U. S. A. 2007, 104:1561924.

53. Eissenberg JC, Elgin SCR: Heterochromatin and Euchromatin In eLS. . John Wiley \& Sons, Ltd; 2001.

54. Crisp M, Liu Q, Roux K, Rattner JB, Shanahan C, Burke B, Stahl PD, Hodzic D: Coupling of the nucleus and cytoplasm: Role of the LINC complex. J. Cell Biol. 2006, 172:41-53.

55. Gundersen GG, Worman HJ: Nuclear positioning. Cell 2013, 152:1376-89.

56. Chang W, Worman HJ, Gundersen GG: Accessorizing and anchoring the LINC complex for multifunctionality. J. Cell Biol. 2015, 208:11-22.

57. Dupin I, Sakamoto Y, Etienne-Manneville S: Cytoplasmic intermediate filaments mediate actin-driven positioning of the nucleus. J. Cell Sci. 2011, 124:865-72.

58. Ketema $M$, Kreft $M$, Secades $P$, Janssen $H$, Sonnenberg A: Nesprin-3 connects plectin and vimentin to the nuclear envelope of Sertoli cells but is not required for Sertoli cell function in spermatogenesis. Mol. Biol. Cell 2013, 24:2454-66.

59. Wu J, Kent I a., Shekhar N, Chancellor TJ, Mendonca A, Dickinson RB, Lele TP: Actomyosin pulls to advance the nucleus in a migrating tissue cell Biophys. $J$. 2014, 106:7-15.

${ }^{* *} 60$. Petrie RJ, Koo H, Yamada KM: Generation of compartmentalized pressure by a 
nuclear piston governs cell motility in a 3D matrix Science 2014, 345:1062-5.

This is the first study to demonstrate that the nucleus can act as a "piston" to drive 3-D cell migration and cytoplasmic protrusion at the leading edge. By measuring the intracellular pressure at the front and back of the migrating cell, the authors show that the nucleus is pulled forward by actomyosin bundles and vimentin filaments, which pressurizes the front compartment to drive lobopodia formation at the leading edge.

61. Tsai J-W, Lian W-N, Kemal S, Kriegstein AR, Vallee RB: Kinesin 3 and cytoplasmic dynein mediate interkinetic nuclear migration in neural stem cells. Nat. Neurosci. 2010, 13:1463-71.

62. Wilson $\mathrm{MH}$, Holzbaur ELF: Opposing microtubule motors drive robust nuclear dynamics in developing muscle cells. J. Cell Sci. 2012, 125:4158-69.

63. Lämmermann T, Bader BL, Monkley SJ, Worbs T, Wedlich-Söldner R, Hirsch K, Keller M, Förster R, Critchley DR, Fässler R, et al.: Rapid leukocyte migration by integrinindependent flowing and squeezing. Nature 2008, 453:51-55.

*64. Thomas DG, Yenepalli A, Denais CM, Rape A, Beach JR, Wang Y-L, Schiemann WP, Baskaran $\mathrm{H}$, Lammerding J, Egelhoff TT: Non-muscle myosin IIB is critical for nuclear translocation during 3D invasion. J. Cell Biol. 2015, 210:583-94.

This is the first study that demonstrates a specific role of NMIIB's in nuclear transloation during 3-D cell migration. Using a microfluidic migration device, traction force microscopy, and collagen invasion assays, the authors show that during 3-D migration, NMIIA is critical for traction force generation at front end of cells, while NMIIB at the back of the cell is crucial for pushing the nucleus through constrictions.

65. Ivkovic S, Beadle C, Noticewala S, Massey SC, Swanson KR, Toro LN, Bresnick AR, Canoll P, Rosenfeld SS: Direct inhibition of myosin II effectively blocks glioma invasion in the presence of multiple motogens. Mol. Biol. Cell 2012, 23:533-42.

66. Stroka KM, Jiang H, Chen S-H, Tong Z, Wirtz D, Sun SX, Konstantopoulos K: Water permeation drives tumor cell migration in confined microenvironments. Cell 2014, 157:611-23.

67. Revach O-Y, Weiner A, Rechav K, Sabanay I, Livne A, Geiger B: Mechanical interplay between invadopodia and the nucleus in cultured cancer cells. Sci. Rep. 2015, 5:9466.

68. Dote H, Burgan WE, Camphausen K, Tofilon PJ: Inhibition of hsp90 compromises the DNA damage response to radiation. Cancer Res. 2006, 66:9211-20.

69. Mayr M, Hu YH, Hainaut $\mathrm{P}, \mathrm{Xu}$ QB: Mechanical stress-induced DNA damage and racp38MAPK signal pathways mediate p53-dependent apoptosis in vascular smooth muscle cells Faseb J. 2002, 16:1423-5.

**70. Guilluy C, Osborne LD, Van LL, Sharek L, Superfine R, Garcia-Mata R, Burridge K: Isolated nuclei adapt to force and reveal a mechanotransduction pathway in the nucleus. Nat.Cell Biol. 2014, 16:376-81.

This is the first study to provide direct evidence that the nucleus itself can act as a mechanosensor. The authors use magnetic beads coated with an anti-nesprin antibody to apply mechanical force to isolated nuclei, while measuring the induced bead displacement and assessing changes in nuclear envelope proteins. The experiments reveal that force application to the nucleus via the LINC complex causes nuclear 
stiffening, phosphorylation of emerin by Src, and recruitment of lamin $A / C$ to the nuclear envelope. These nuclear mechanotransduction processes require lamin $A / C$ and emerin, but are independent of chromatin and nuclear actin rearrangments.

71. Li Y, Chu JS, Kurpinski K, Li X, Bautista DM, Yang L, Paul Sung KL, Li S: Biophysical regulation of histone acetylation in mesenchymal stem cells Biophys. J. 2011, 100:1902-9.

72. lyer KV, Pulford S, Mogilner a., Shivashankar G V.: Mechanical activation of cells induces chromatin remodeling preceding MKL nuclear transport Biophys. J. 2012, 103:1416-28.

73. Poh Y-C, Shevtsov SP, Chowdhury F, Wu DC, Na S, Dundr M, Wang N: Dynamic forceinduced direct dissociation of protein complexes in a nuclear body in living cells. Nat. Commun. 2012, 3:866.

74. Kaminski A, Fedorchak GR, Lammerding J: The cellular mastermind(?)mechanotransduction and the nucleus. Prog. Mol. Biol. Transl. Sci. 2014, 126:157203.

75. Guilluy C, Burridge K: Nuclear mechanotransduction: forcing the nucleus to respond. Nucleus 2015, 6:19-22.

76. Cost A-L, Ringer P, Chrostek-Grashoff A, Grashoff C: How to Measure Molecular Forces in Cells: A Guide to Evaluating Genetically-Encoded FRET-Based Tension Sensors Cell. Mol. Bioeng. 2015, 8:96-105.

77. Wolf K, Alexander S, Schacht V, Coussens LM, von Andrian UH, van Rheenen J, Deryugina $\mathrm{E}$, Friedl $\mathrm{P}$ : Collagen-based cell migration models in vitro and in vivo. Semin. Cell Dev. Biol. 2009, 20:931-41.

78. Haeger A, Krause M, Wolf K, Friedl P: Cell jamming: Collective invasion of mesenchymal tumor cells imposed by tissue confinement. Biochim. Biophys. Acta Gen. Subj. 2014, 1840:2386-95.

79. Meehan S, Nain AS: Role of Suspended Fiber Structural Stiffness and Curvature on Single-Cell Migration, Nucleus Shape, and Focal-Adhesion-Cluster Length. Biophys. J. 2014, 107:2604-11.

80. Qin S, Ricotta V, Simon M, Clark R a. F, Rafailovich MH: Continual Cell Deformation Induced via Attachment to Oriented Fibers Enhances Fibroblast Cell Migration. PLoS One 2015, 10:e0119094.

81. Duncombe TA, Tentori AM, Herr AE: Microfluidics: reframing biological enquiry. Nat. Rev. Mol. Cell Biol. 2015, 16:554-67.

82. Lautenschläger F, Piel M: Microfabricated devices for cell biology: all for one and one for all. Curr. Opin. Cell Biol. 2013, 25:116-24.

83. Lange JR, Steinwachs J, Kolb T, Lautscham LA, Harder I, Whyte G, Fabry B: Microconstriction Arrays for High-Throughput Quantitative Measurements of Cell Mechanical Properties. Biophys. J. 2015, 109:26-34.

84. Schreiber KH, Kennedy BK: When Lamins Go Bad: Nuclear Structure and Disease. Cell 2013, 152:1365-75.

*85. Davidson PM, Lammerding J: Broken nuclei - lamins, nuclear mechanics, and 
disease. Trends Cell Biol. 2014, 24:247-56.

This review provides a concise overview of the importance of lamins in health and disease, integrating lamin's various roles on nuclear structure, mechanotransduction signaling, and gene regulation.

86. Krause M, Wolf K: Cancer cell migration in 3D tissue: Negotiating space by proteolysis and nuclear deformability. Cell Adh. Migr. 2015, 9:357-66.

87. Denais C, Lammerding J: Nuclear mechanics in cancer. Adv. Exp. Med. Biol. 2014, 773:435-70.

88. Capo-chichi CD, Cai KQ, Smedberg J, Azar PG-A, Godwin AK, Xu X-X: Original Article Materials and Methods Tumor specimens, human primary Small interfering RNA transfection ( shRNA and siRNA ) Flow cytometry analysis Probes for Northern blot and antibodies for. Chin. J. Cancer 2011, 30:415-25.

89. Wazir U, Ahmed MH, Bridger JM, Harvey A, Jiang WG, Sharma AK, Mokbel K: The clinicopathological significance of lamin A/C, lamin B1 and lamin B receptor mRNA expression in human breast cancer. Cell. Mol. Biol. Lett. 2013, 18:595-611.

90. Capo-chichi CD, Aguida B, Chabi NW, Cai QK, Offrin G, Agossou VK, Sanni A, Xu X-X: Lamin A/C deficiency is an independent risk factor for cervical cancer. Cell. Oncol. 2015, doi:10.1007/s13402-015-0252-6.

91. Belt EJT, Fijneman RJA, van den Berg EG, Bril H, Delis-van Diemen PM, Tijssen M, van Essen HF, de Lange-de Klerk ESM, Beliën JAM, Stockmann HBAC, et al.: Loss of lamin A/C expression in stage II and III colon cancer is associated with disease recurrence. Eur. J. Cancer 2011, 47:1837-45.

92. Willis ND, Cox TR, Rahman-Casañs SF, Smits K, Przyborski SA, van den Brandt P, van Engeland $M$, Weijenberg $M$, Wilson RG, de Bruïne $A$, et al.: Lamin $\mathbf{A} / \mathbf{C}$ is a risk biomarker in colorectal cancer. PLoS One 2008, 3:e2988.

93. Chow K-H, Factor RE, Ullman KS: The nuclear envelope environment and its cancer connections. Nat. Rev. Cancer 2012, 12:196-209.

94. Mitchell MJ, Denais C, Chan MF, Wang Z, Lammerding J, King MR: Lamin A/C deficiency reduces circulating tumor cell resistance to fluid shear stress $A m$. J. Physiol. - Cell Physiol. 2015, 309:C736-46.

95. Chiotaki R, Polioudaki H, Theodoropoulos $\mathrm{P}$ a: Differential nuclear shape dynamics of invasive and non-invasive breast cancer cells are associated with actin cytoskeleton organization and stability. 2014, 295:287-95.

96. Jain N, lyer KV, Kumar A, Shivashankar G V: Cell geometric constraints induce modular gene-expression patterns via redistribution of HDAC3 regulated by actomyosin contractility. Proc. Natl. Acad. Sci. U. S. A. 2013, 110:11349-54.

97. Meuleman W, Peric-Hupkes D, Kind J, Beaudry JB, Pagie L, Kellis M, Reinders M, Wessels L, Van Steensel B: Constitutive nuclear lamina-genome interactions are highly conserved and associated with A/T-rich sequence. Genome Res. 2013, 23:270-80.

98. Petrie RJ, Yamada KM: Fibroblasts Lead the Way: A Unified View of 3D Cell Motility Trends Cell Biol. 2015, 25:666-74. 
99. Petrie RJ, Gavara N, Chadwick RS, Yamada KM: Nonpolarized signaling reveals two distinct modes of 3D cell migration. J. Cell Biol. 2012, 197:439-55.

100. Levy JR, Holzbaur ELF: Dynein drives nuclear rotation during forward progression of motile fibroblasts. J. Cell Sci. 2008, 121:3187-95.

101. Sosa B a., Rothballer A, Kutay U, Schwartz TU: LINC complexes form by binding of three KASH peptides to domain interfaces of trimeric SUN proteins. Cell 2012, 149:1035-47. 
(A)
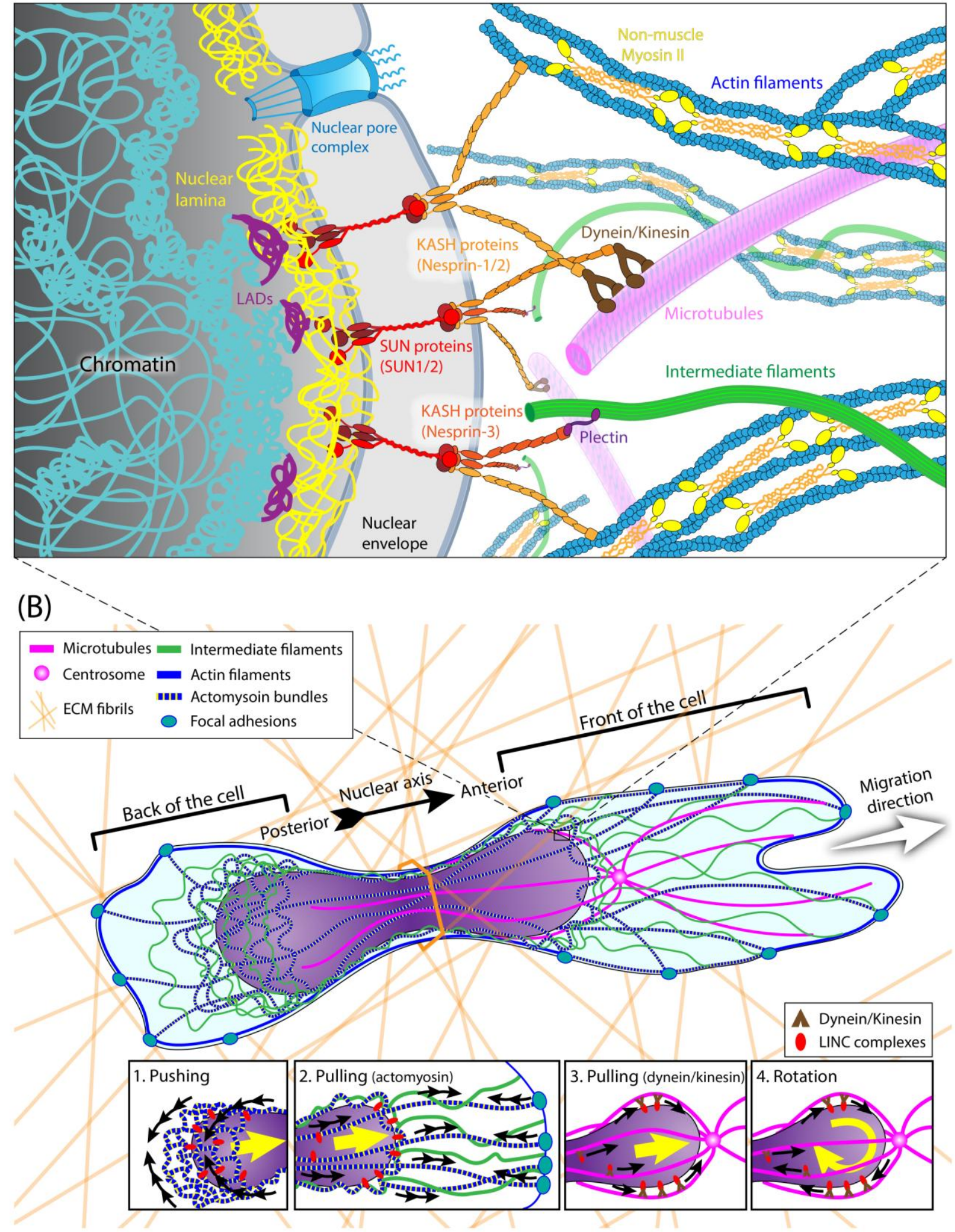

Figure 1 